\title{
Belgeo
}

Revue belge de géographie

$1 \mid 2014$

From urban renewal to metropolitan strategies?

Cultural flagship projects in restructuring industrial areas

\section{Vandermotten C., Bruxelles, une lecture de la ville. De L'Europe des marchands à la capitale de l'Europe}

Editions de l'Université de Bruxelles, Collection UBlire, 2014, 233 pages

\section{Mathieu Van Criekingen}

\section{OpenEdition}

\section{Journals}

Édition électronique

URL : http://journals.openedition.org/belgeo/12840

DOI : 10.4000/belgeo.12840

ISSN : 2294-9135

Éditeur :

National Committee of Geography of Belgium, Société Royale Belge de Géographie

Référence électronique

Mathieu Van Criekingen, « Vandermotten C., Bruxelles, une lecture de la ville. De L'Europe des marchands à la capitale de l'Europe », Belgeo [En ligne], 1 | 2014, mis en ligne le 15 décembre 2014, consulté le 22 septembre 2020. URL : http://journals.openedition.org/belgeo/12840; DOI : https://doi.org/10.4000/ belgeo. 12840

Ce document a été généré automatiquement le 22 septembre 2020.

Belgeo est mis à disposition selon les termes de la licence Creative Commons Attribution 4.0 International. 


\title{
Vandermotten C., Bruxelles, une lecture de la ville. De L'Europe des marchands à la capitale de l'Europe
}

Editions de l'Université de Bruxelles, Collection UBlire, 2014, 233 pages

\author{
Mathieu Van Criekingen
}

\section{RÉFÉRENCE}

Vandermotten C., Bruxelles, une lecture de la ville. De L'Europe des marchands à la capitale de l'Europe, Editions de l'Université de Bruxelles, Collection UBlire, 233 pages

1 L'ouvrage de Christian Vandermotten annonce d'emblée son ambition: faire comprendre Bruxelles. Aux yeux du géographe, il s'agit de comprendre comment les paysages bâtis se mettent en place, comment les divisions sociales et fonctionnelles de l'espace urbain s'installent, se reproduisent et se transforment, ou comment, encore, un territoire métropolitain se construit.

2 L'auteur déploie à cette fin une grille de lecture particulière, inspirée de la géographie radicale, qui montre ici toute sa pertinence et son efficacité. Au fondement de cette " conception matérialiste de la production de l'espace urbain» (p. 219) (voir aussi Vandermotten, 2008), figure l'idée simple mais essentielle que l'espace urbain est d'abord une construction sociale. Comprendre la ville demande, dès lors, de se pencher sur les rapports de force sociaux et politiques qui «fabriquent " ses paysages comme les partitions sociales et démographiques de son territoire. Cette lecture est ici inscrite dans une perspective historique de long terme, donnant toute leur place aux processus de reproduction des structures socio-spatiales héritées, aux côtés des dynamiques de transformation.

On l'aura compris, la lecture proposée par Christian Vandermotten, se distingue totalement des lectures aujourd'hui les plus en vue des «questions urbaines » dans le champ politico-médiatique : ici, aucune métaphore sur les « mutations » (naturelles) du 
«corps urbain" ni écho aux concerts d'enthousiasmes sur l'avènement de "villes intelligentes ". Ce point n'est pas secondaire dans la mesure où cet ouvrage n'entend pas s'adresser qu'à un public académique spécialisé. Plutôt, et c'est là un tour de force, l'auteur parvient à faire efficacement tenir son propos dans un format de poche d'un peu plus de 200 pages, riche de 62 figures (dont de nombreuses cartes), de 16 tableaux et de listes bibliographiques thématiques et commentées, présentées en fin de chacun des cinq chapitres.

4 Cette lecture est déployée tout au long de l'ouvrage à trois échelles: des formes architecturales d'édifices emblématiques - La Grand Place, par exemple (échelle micro), aux configurations d'ensembles urbains - comme le quartier Léopold devenu district européen, par exemple (échelle méso), jusqu'aux configurations de l'agglomération dans son ensemble en lien avec la structuration de l'espace belge comme avec le positionnement de Bruxelles dans les hiérarchies urbaines européennes et mondiales (échelle macro).

$5 \quad$ C'est dans les chapitres 4 et 5 (respectivement : «La formation de l'espace et des paysages urbains, des origines à la mise en place de la Région de Bruxelles-Capitale » et « Aménagement, projets de développement et rapports de force politiques dans le Bruxelles post-fordiste ») que cette grille de lecture est déployée le plus directement. Les trois premiers chapitres fixent, eux, les éléments de contextualisation nécessaires. L'auteur y revient notamment sur la question des limites de Bruxelles et des découpages politicoadministratifs de l'agglomération (soulevant entre autres la question des concurrences internes entre centre et périphérie), sur le profil particulier de Bruxelles dans le concert des villes globales, sur les liens entre performances de l'économie métropolitaine et creusement des inégalités sociales, ou encore sur les questions démographiques et linguistiques.

6 Christian Vandermotten propose, en conclusion, un précieux tableau synoptique des « forces sociales et politiques urbaines du milieu du XIXème siècle à aujourd'hui » (p. 222), fruit de sa lecture historicisée de la production de l'espace bruxellois. Outre sa valeur pédagogique, cette synthèse permet de pointer l'importance politique du positionnement des classes intermédiaires dans les évolutions des orientations dominantes en matière d'aménagement et de développement urbains. Les choix résidentiels, les modes de consommation comme les « visions " politiques de ces classes intermédiaires - elles-mêmes fractionnées - pèsent en effet d'un grand poids sur les formes matérielles et symboliques de la fabrique de la ville. Or, «(...) aujourd'hui, on assiste à un nouveau glissement: abandonnant la position défensive qu'elles avaient adoptée durant les deux dernières décennies du siècle dernier, les classes moyennes aisées sont passés à une stratégie de réappropriation [des quartiers centraux], valorisant l'internationalisation cosmopolite, qui conforte aussi leur patrimoine immobilier. Ceci les rapproche de la promotion immobilière. D'autant que celle-ci tient désormais un discours post-moderne esthétisant et de développement durable, avec une vision plus intégrée, plus diversifiée, qui ne néglige plus le résidentiel (aisé). Les positions [du Gouvernement] de la Région s'appuient sur cette nouvelle coalition" (p. 224). Dans une ville où les quartiers centraux sont, pour partie importante, des quartiers populaires et d'accueil des populations migrantes, cette question des alliances de (fractions de) classes autour d'un «projet de ville » qui vise à une « réappropriation » de ces quartiers est très lourde d'enjeux sociaux et politiques. 


\section{BIBLIOGRAPHIE}

Vandermotten C. (2008), « Géographie et production de l'espace : réflexions épistémologiques sur les rapports entre une science et la société », Geographica Helvetica, 4, pp. 228-236. 